\title{
Ecology of galaxy stellar populations from optical spectroscopic surveys
}

\author{
Anna Gallazzi ${ }^{1}$ \\ ${ }^{1}$ Max-Planck-Institut für Astronomie, Königstuhl 17, 69117, Heidelberg, Germany \\ email: gallazzi@mpia.de
}

\begin{abstract}
The age and chemical composition of the stars in present-day galaxies carry important clues about their star formation processes. The latest generation of population synthesis models have allowed to derive age and stellar metallicity estimates for large samples of lowredshift galaxies. After reviewing the main results about the distribution in ages and metallicities as a function of galaxy mass, I will concentrate on recent analysis that aims at disentangling the dependences of stellar populations properties on environment and on galaxy stellar mass. Finally, new models that predict the response of the full spectrum to variations in $[\alpha / \mathrm{Fe}]$ will allow us to derive accurate estimates of elements abundance ratios and gain deeper insight into the timescales of star formation cessation.
\end{abstract}

Keywords. galaxies: evolution, galaxies: formation, galaxies: fundamental parameters, surveys

\section{Introduction}

The age and chemical composition of the stellar populations in galaxies, together with galaxy mass, are key ingredients to uncover galaxy formation and evolutionary paths. Estimates of stellar populations parameters are derived by interpreting detailed spectral information, such as absorption features, on the basis of stellar population synthesis (SPS) models. Our ability of interpreting galaxy spectra has greatly improved with the development of SPS models that i) predict the full spectrum of simple stellar populations (SSPs) at medium/high resolution, allowing to adjust the models to the data quality rather than the other way round, and ii) have a better coverage of the stellar parameters space, allowing the interpretation of a broader range of stellar populations (Vazdekis 1999; Bruzual \& Charlot 2003; Coelho et al. 2007).

Together with the development of SPS models and spectral fitting techniques, the statistical power of large spectroscopic surveys has allowed to put on a firm ground our understanding of the stellar populations in nearby galaxies. In this contribution I will briefly review results on the dependence of stellar populations properties on galaxy mass, as obtained from the analysis of Sloan Digital Sky Survey (SDSS) galaxy spectra. I will then discuss to what extent these relations are shaped by the environment in which galaxies reside, as recently analysed in Pasquali et al. (2009, submitted) combining the SDSS DR4 catalogue of stellar ages and metallicities with the Yang et al. (2007) group catalogue. I will conclude with a brief outlook on new population synthesis models that allow accurate estimates of $\alpha$-elements abundance ratios.

\section{The ages and metallicities of present-day stellar populations}

\subsection{Estimates of stellar populations parameters}

Estimates of stellar metallicity, luminosity-weighted age and stellar mass have been derived for the spectroscopic sample of SDSS DR4 by interpreting the strength of a set of 
absorption features including the $4000 \AA$ break, the Balmer lines and $[\alpha / \mathrm{Fe}]$-insensitive $\mathrm{Mg}$ and Fe composite indices. A library of galaxy spectral features is generated by convolving Bruzual \& Charlot (2003) SSPs with a comprehensive set of Monte Carlo star formation histories (SFHs). Following a Bayesian statistical approach, the likelihood distribution of each parameter has been computed by comparing the observed absorption features with those predicted by the full library.

The uncertainties on physical parameters estimates depend on the spectral signal-tonoise $(\mathrm{S} / \mathrm{N})$ and on the galaxy spectral type or SFH. A careful analysis of the statistical uncertainties on stellar $\mathrm{M} / \mathrm{L}$, arguably the easiest parameter to constrain, indicates two interesting points for model development and spectral analysis: i) for galaxies with old stellar populations and spectra of good $\mathrm{S} / \mathrm{N}$ statistical uncertainties of 0.03 dex could in principle be reached, indicating the need for continuous model development and control of systematics; ii) on the other hand for galaxies with recent bursts of star formation the uncertainties are limited by the large intrinsic scatter in physical parameters at fixed index strength: it is thus crucial to properly model the complexity of SFHs in the Universe (Gallazzi \& Bell 2009, submitted).

\subsection{Dependence on stellar mass}

In our previous studies, based on a sample of SDSS DR2 galaxies at $z<0.22$, we have analysed the distribution of galaxies in stellar metallicity-age-stellar mass. In the mean, low-mass galaxies are young and metal-poor while high-mass galaxies are old and metal-rich. There is a rapid transition between these two regimes at stellar masses around $10^{10.5} M_{\odot}$ (Gallazzi et al. 2005). This corresponds to the transition regime in other galaxy properties (e.g., Kauffmann et al. 2003; Baldry et al. 2004), and it is where the scatter in stellar populations parameters is largest.

Early-type galaxies, which contain the majority of baryons and metals in stars today (Gallazzi et al. 2008), dominate above the transition mass where the relations between metallicity/age and mass flattens. They follow a tight color-magnitude relation (CMR) which is driven by an increase with stellar mass of both stellar age, total metallicity and elements abundance ratios (Gallazzi et al. 2006), suggesting that the stars in massive early-type galaxies have formed earlier and on shorter timescales than less massive ones.

\subsection{Dependence on environment: centrals versus satellites}

The bottom line of the above results is that galaxy $\mathrm{SFH}$ is primarily determined by galaxy mass. The scatter in physical parameters at fixed mass, however, indicates underlying second-order dependences. The star formation activity in galaxies is also sensitive to the environment in which galaxies reside, as witnessed by the SFR-density relation (e.g., Lewis et al. 2002) and by the differences in scatter and zero-point of the CMR of earlytype galaxies in different environments (e.g., Thomas et al. 2005; Gallazzi et al. 2006). These environmental dependences are however subtle, once the primary dependence on stellar mass is removed. Moreover the signal may depend on the environmental estimator adopted, such as e.g. galaxy number densities in apertures which have the disadvantage of being sensitive to environment itself and not directly comparable to the more physical description in terms of host dark matter halos of galaxy formation models.

In Pasquali et al. (2009) we take advantage of the group catalogue by Yang et al. (2007) which assign a dark matter mass to galaxy groups and classify their members into centrals and satellites. We complement the galaxy group catalogue with the stellar metallicity and age estimates computed for SDSS DR4. We then analyse the dependence of stellar age and metallicity on stellar mass, distinguishing centrals and satellites and as a function of host halo mass. 

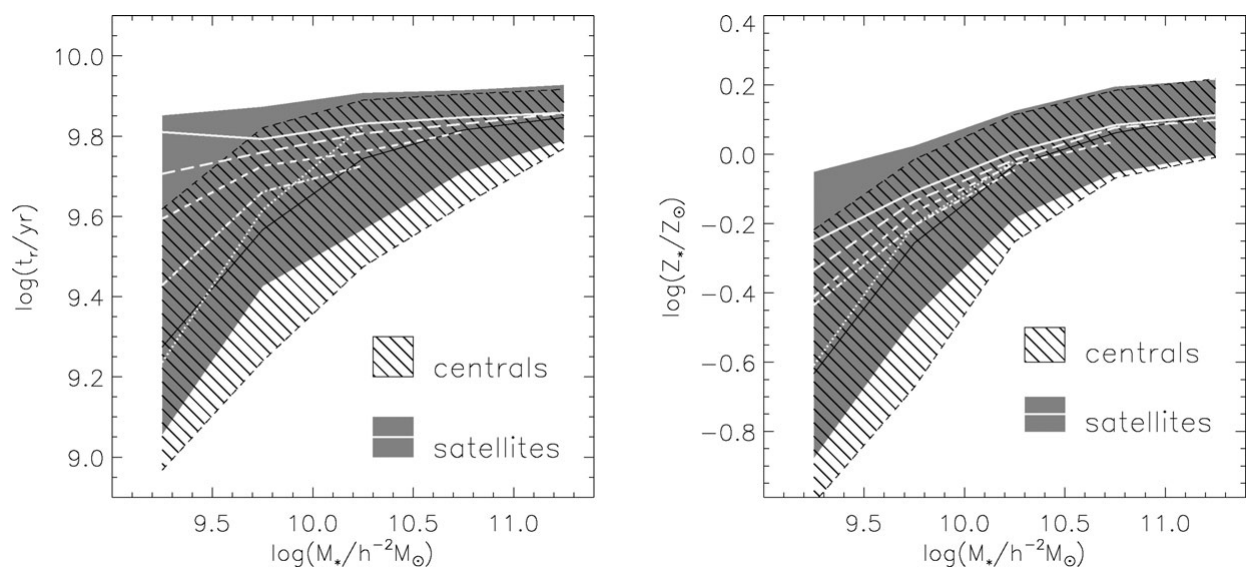

Figure 1. Luminosity-weighted age (Left) and stellar metallicity (Right) as a function of stellar mass. The full $16 \%-84 \%$ range of the distributions is shown. Galaxies are separated into centrals (hatched region) and satellites (grey shaded region). The median relations for satellites galaxies hosted by halos of different mass are shown by the white lines $\left(\mathrm{M}_{\mathrm{halo}} / \mathrm{h}^{-1} \mathrm{M}_{\odot}\right.$ : $14-15$ (solid), 13-14 (long dashed), 12.5-13 (dashed), 12-12.5 (dot-dashed), 11-12 (dotted)). Figure adapted from Pasquali et al. (2009, submitted).

The left panel of Fig. 1 shows the relations between luminosity-weighted age and stellar mass for central (hatched region) and satellite galaxies (grey shaded region). The relations between stellar metallicity and mass are shown in the right-hand panel. At stellar masses $M_{*}>10^{10.5} M_{\odot}$ centrals and satellites have the same mean ages and metallicities. At lower stellar masses instead, satellite galaxies are older and metal-richer than equally-massive centrals. Moreover, we note in the satellite population an excess of old, metal-rich galaxies at low $\mathrm{M}_{*}$ and a lack of young metal-poor galaxies at nearly all masses. We reproduce the result found in other studies that at all scales, centrals are older and metal-richer than satellites that live in equally-massive halos. This likely reflects the general trends of age/metallicity with mass and the fact that in a given halo centrals are more massive than satellites. We also find that, while the stellar populations of central galaxies are nearly independent of halo mass, this is not the case for satellite galaxies. The white lines of different style in Fig. 1 show the age/metallicity-mass relations for satellite galaxies in halos of different mass. Below $M_{*} \sim 10^{10.5} M_{\odot}$, at fixed stellar mass the stellar populations of satellite galaxies depend on halo mass: satellites in more massive halos are older and metal-richer than those in less massive halos.

At high masses, the SFH of galaxies is likely regulated by internal feedback processes regardless of environment. At low masses instead the SFH of satellite galaxies is affected by environmental processes, whereby their stellar populations differ from those of equallymassive centrals by an amount that increases with halo mass.

\section{Elements abundance ratios}

The results discussed above indicate that satellite galaxies are affected by the environment onto which they are accreted through mechanisms that quench star formation and prevent further replenishment of gas via inflows. It remains to be determined on which timescale the SF in satellite galaxies is quenched and any possible dependence on halo mass. Important clues about SF timescales can be gained from the abundance of $\alpha$-elements with respect to $\mathrm{Fe}([\alpha / \mathrm{Fe}])$. Previous results suggest that the $[\alpha / \mathrm{Fe}]$ of isolated early-type galaxies is the same as for cluster galaxies (Thomas et al. 2005). A 
careful definition of environment (such as the one introduced in the previous section) and accurate measures of $[\alpha / \mathrm{Fe}]$ are necessary to confirm or disprove these findings.

Theoretical SPS models that predict the full spectrum of stellar populations with different $[\alpha / \mathrm{Fe}]$ are now available (Coelho et al. 2007). Recently, Walcher et al. (2009) have developed 'differential' stellar populations models that exploit the predictive power of fully theoretical models, calibrated onto semi-empirical solar metallicity SSPs. In this way it is possible to predict the pixel-by-pixel absolute flux (as opposed to absorption indices only) as a function of both $[\mathrm{Fe} / \mathrm{H}]$ and $[\alpha / \mathrm{Fe}]$.

We are now in a position to predict spectra for stellar populations with variable $[\alpha / \mathrm{Fe}]$ (in addition to metallicity) and with complex SFHs, and exploit the high resolution of the models to: i) convolve them to match the effective resolution of the data (including velocity dispersion broadening), ii) use the redundancy of the full spectral information for low-quality data, in addition to individual absorption indices.

We have generated a Monte Carlo library of SFHs based on the new differential models. Adopting the same Bayesian approach used before (see Section 2.1) we can derive consistent estimates of $\alpha$-elements abundance ratio together with luminosity-weighted age, metallicity and mass-to-light ratios, and their associated uncertainties. The analysis of a well-defined sample of early-type galaxies will enable us to validate the model predictions and test the sensitivity of total metallicity and stellar $\mathrm{M} / \mathrm{L}$ to $[\alpha / \mathrm{Fe}]$ variations.

Estimates of elements abundance ratios for large samples of both early-type and starforming galaxies (exploiting the better coverage of the models to high temperatures) will allows us to address several key question on SF timescale in galaxies.

\section{Acknowledgements}

I would like to thank in particular Anna Pasquali and Jakob Walcher for their substantial contribution to the work presented here. I am grateful to many colleagues for their support in different stages of this research: Stéphane Charlot, Jarle Brinchmann, Paula Coelho, Gustavo Bruzual, Eric Bell and Simon White.

\section{References}

Baldry, I. K., Glazebrook, K., Brinkmann, J., Ivezić, Ž., Lupton, R. H., Nichol, R. C., \& Szalay, A. S. 2004, ApJ, 600, 681

Bruzual, G. \& Charlot, S. 2003, MNRAS, 344, 1000

Coelho, P., Bruzual, G., Charlot, S., Weiss, A., Barbuy, B., \& Ferguson, J. W. 2007, MNRAS, 382,498

Gallazzi, A. \& Bell, E. F. 2009, ApJS, submitted

Gallazzi, A., Brinchmann, J., Charlot, S., \& White, S. D. M. 2008, MNRAS, 383, 1439

Gallazzi, A., Charlot, S., Brinchmann, J., \& White, S. D. M. 2006, MNRAS, 370, 1106

Gallazzi, A., Charlot, S., Brinchmann, J., White, S. D. M., \& Tremonti, C. A. 2005, MNRAS, 362,41

Kauffmann, G. et al. 2003, MNRAS, 341, 54

Lewis, I. et al. 2002, MNRAS, 334, 673

Pasquali, A., Gallazzi, A., Fontanot, F., van den Bosch, F. C., de Lucia, G., Mo, H. J., \& Yang, X. 2009, MNRAS, submitted

Thomas, D., Maraston, C., Bender, R., \& Mendes de Oliveira, C. 2005, ApJ, 621, 673

Vazdekis, A. 1999, ApJ, 513, 224

Yang, X., Mo, H. J., van den Bosch, F. C., Pasquali, A., Li, C., \& Barden, M. 2007, ApJ, 671, 153

Walcher, C. J., Coelho, P., Gallazzi, A., \& Charlot, S. 2009, MNRAS, 398, L44 